Pacific Journal of Mathematics

LARGE SUBLATTICES OF A LATTICE 


\section{LARGE SUBLATTICES OF A LATTICE}

\section{Thomas P. Whaley}

In this paper we consider a special case of the following two closely related problems of $B$. Jónsson:

I. For which infinite cardinals $m$ is there an algebra of power $m$ which has finitely many operations and satisfies the descending chain condition for subalgebras.

II. For which infinite cardinals $m$ is there an algebra of power $m$ which has finitely many operations and has no proper subalgebra of power $m$.

Of course a positive answer to the first problem for a given cardinal always indicates a positive answer to the second for the same cardinal.

The special case we are concerned with is obtained by further restricting the algebras to be lattices. With this restriction we obtain a negative answer to the second problem for any regular cardinal. It follows that the answer to the first question is negative for the class of lattices and for any infinite cardinal $m$. Actually we obtain a stronger result which shows that in a lattice of power $m$ where $m$ is infinite and regular, there are at most two elements which do not lie in the complement of a sublattice of power $m$. We give an example to show that regularity is needed here. In a distributive lattice of regular cardinality $m$ every element lies in the complement of some sublattice of cardinality $m$.

We adopt the conventions of identifying an ordinal with the set of smaller ordinals and of identifying a cardinal $m$ with the smallest ordinal of cardinality $m$.

The bibliography includes most of the results related to problems I and II.

2. Lattices of regular cardinality. Throughout this paper $\langle L ;+, \cdot, \leqq\rangle$ will denote a lattice of power $m \geqq \omega$ in which $x+y$ is the least upper bound of $\{x, y\}$ and $x \cdot y$ is the greatest lower bound of $\{x, y\}$ for any $x, y \in L$. We usually identify such a lattice with the underlying set $L$. We use the notation $K \subseteq_{s} L$ to indicate that $K$ is a sublattice of $L$. For $x, y \in L$ we let

$$
S(x, y)=\{z \in L \mid z+x=y\}
$$

and

$$
T(x, y)=\{z \in L \mid z \cdot x=y\}
$$


In particular $S(x, x)$ is the principal ideal generated by $x$ and $T(x, x)$ is the principal dual ideal generated by $x$. We write $S(x)$ for $S(x, x)$ and $T(x)$ for $T(x, x)$. Thus for any $x \in L, S(x) \subseteq_{s} L$ and $T(x) \subseteq_{s} L$. It is easy to check that $S(x, y)$ and $T(x, y)$ are sublattices of $L$ for any $x, y \in L$ if $L$ is distributive. Since for any $x, y \in L$ we have $x+y \in T(x)$ and $y \in S(x, x+y)$, we see that

(1) $L=\bigcup\{S(x, z) \mid z \in T(x)\}$

disjointly and dually

(2) $L=\bigcup\{T(x, z) \mid z \in S(x)\}$

disjointly.

If $k$ is a cardinal, we let $M_{k}$ be the two-dimensional lattice having $k$ atoms. We indicate this lattice in our diagrams by the figure

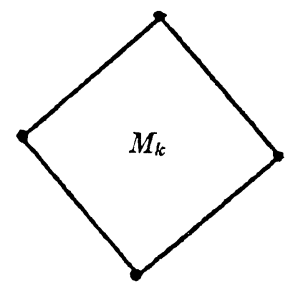

FIGURE 1.

If $L$ has a largest element, we denote this element by 1 , and we let 0 indicate the smallest element of $L$ if such an element exists. If $L$ has a largest and a smallest element and $x \in L$, we let $C(x)$ be the set of all complements of $x$, i.e., $C(x)=S(x, 1) \cap T(x, 0)$.

The following theorem yields some immediate results concerning the problems under investigation. This theorem is also used in almost every proof of this paper.

Theorem 2.1. If $L$ is a lattice of power $m$ where $m$ is infinite and regular, then one of the following conditions must hold:

(i ) There is an $x \in L$, distinst from 1 if 1 exists, with $|S(x)|=m$.

(ii) There is an $x \in L$, distinst from 0 if 0 exists, with $|T(x)|=m$.

(iii) $L$ has a sublattice isomorphic to $M_{m}$.

Proof. Suppose (i) and (ii) fail. Let $x$ be any element of $L$ other than a possible largest or smallest element. Since (i) and (ii) fail, we have $|S(x) \cup T(x)|<m$. By (1) we have $L=\bigcup\{S(x, y) \mid y \in T(x)\}$ disjointly. Thus $m=|L|=\sum(|S(x, y)| ; y \in T(x))$. Since $|T(x)|<m$ and $m$ is regular, there is a $y_{0} \in T(x)$ with $\left|S\left(x, y_{0}\right)\right|=m$. It is clear that $S\left(x, y_{0}\right) \subseteq S\left(y_{0}\right)$. Hence $\left|S\left(y_{0}\right)\right|=m$ implying that $y_{0}=1$. If $y \in T(x)-\{1\}$, then $|S(y)|<m$ so $|S(x, y)|<m$. Thus 


$$
\begin{aligned}
|L-S(x, 1)| & =|\bigcup\{S(x, y) \mid y \in T(x)-\{1\}\}| \\
& =\sum(|S(x, y)| ; y \in T(x)-\{1\}) \\
& <m .
\end{aligned}
$$

Similarly, $|L-T(x, 0)|<m$. Combining these inequalities, we get

$$
\begin{aligned}
|L-C(x)| & =|L-(S(x, 1) \cap T(x, 0))| \\
& \leqq|L-S(x, 1)|+|L-T(x, 0)| \\
& <m .
\end{aligned}
$$

This holds for any $x \neq 0,1$. We use this fact to obtain a sequence $\left\{x_{\xi} \mid \xi<m\right\} \subseteq L-\{0,1\}$ so that $x_{\xi} \in C\left(x_{\xi^{\prime}}\right)$ whenever $\xi, \xi^{\prime}<m$ and $\xi \neq \xi^{\prime}$. Inductively, suppose we have $\beta<m$ and $\left\{x_{\xi} \mid \xi<\beta\right\}$ with this property. Note that

$$
\begin{aligned}
\left|L-\bigcap\left\{C\left(x_{\xi}\right) \mid \xi<\beta\right\}\right| & =\left|\bigcup\left\{\left(L-C\left(x_{\xi}\right)\right) \mid \xi<\beta\right\}\right| \\
& \leqq \sum\left(\left|L-C\left(x_{\xi}\right)\right| ; \xi<\beta\right) \\
& <m
\end{aligned}
$$

since $m$ is regular, $\beta<m$, and for each $\xi<\beta$, $\left|L-C\left(x_{\xi}\right)\right|<m$. Now we take $x_{\beta} \in \bigcap\left\{C\left(x_{\xi}\right) \mid \xi<\beta\right\}$. Clearly $\left\{x_{\xi} \mid \xi<m\right\} \bigcup\{0,1\}$ is a sublattice of $L$ isomorphic to $M_{m}$.

CoROLlaRY 2.2. If $L$ is a lattice of cardinality $m$ where $m$ is infinite and regular, then $L$ has a proper sublattice of power $m$.

CoRollary 2.3. No infinite lattice satisfies the descending chain condition for subalgebras.

COROLlaRY 2.4. If $L$ is a lattice of cardinality $m$ where $m$ is infinite and regular, then one of the following must hold:

(i) $L$ has an infinite chain of elements

(ii) $L$ has a sublattice isomorphic to $M_{m}$.

Proof. Suppose (ii) fails. We obtain (i) by repeatedly applying (i) and (ii) of Theorem 2.1.

CoRollary 2.5. (Well-known). No infinite distributive lattice is finite dimensional.

EXAMPle 2.6. Suppose $m$ is not regular, say $m=\sum\left(m_{\alpha} \mid \alpha \in k\right)$ where each $m_{\alpha}<m$ and $k<m$. Consider the lattice. 


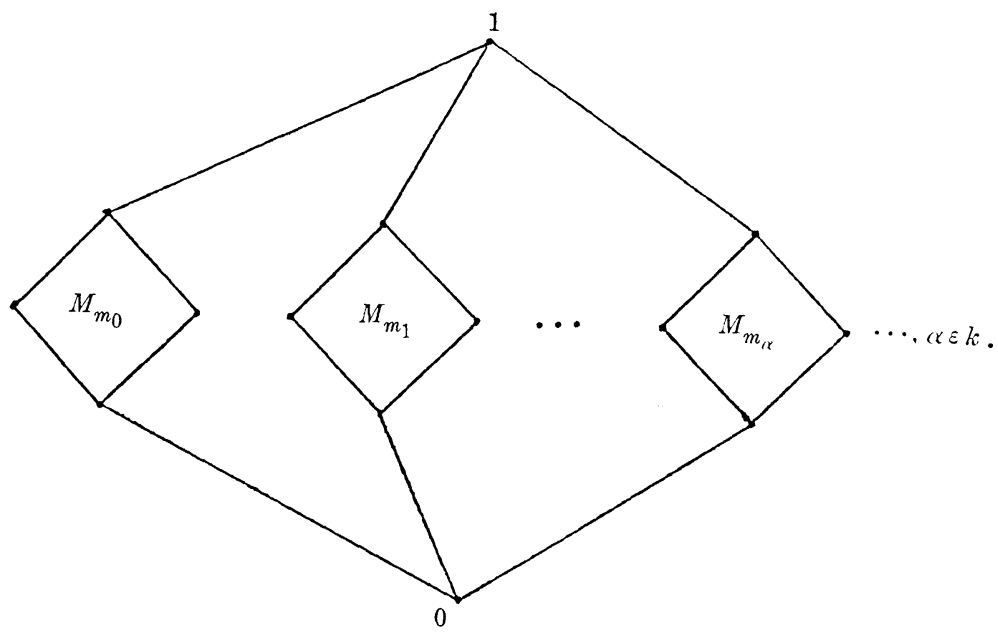

Figure 2

It is clear that (i), (ii), and (iii) of Theorem 2.1 fail in this lattice.

3. Separation of elements by large sublattices. Theorem 2.1 leads us to consider how many elements of a lattice of regular cardinality are disjoint from some "large" sublattice. Also, given two elements of such a lattice, is there a "large" sublattice which contains one but not the other? Of course we note that $M_{k}$ where $k$ is infinite has two elements, 0 and 1 , which are in every "large" sublattice and hence may not be so separated. We proceed now to show that this is essentially the only such example.

Definition 3.1. Suppose $L$ is a lattice of power $m \geqq \omega$ and $K \subseteq_{s} L$ with $|K|=m$. We say $K$ separates $x$ from $y$ if $x \in K$ and $y \in L-K$. We say $x$ can be separated from $y$ if such a $K$ exists.

LEMMA 3.2. Suppose $L$ is a lattice of power $m \geqq \omega$ with $m$ regular. If $y \in L$ has the properties

(i ) $|S(y) \cup T(y)|<m$,

(ii) if $z \in L$ with $|S(z)|=m$, then $y \in S(z)$,

(iii) if $z \in L$ with $|T(z)|=m$, then $y \in T(z)$, then $L$ has a sublattice isomorphic to $M_{m}$ having $y$ as an atom.

Proof. As in the proof of Theorem 2.1 there is a $c \in L$ with $|S(y, c)|=m$. Consider any $z \in S(y, c), z \neq c$. By (ii) and (iii) we have $|S(z) \cup T(z)|<m$. Now

$$
S(y, c)=\bigcup\{S(z, x) \cap S(y, c) \mid x \in T(z)\} .
$$

Thus there is $c^{\prime} \in L$ with $\left|S\left(z, c^{\prime}\right) \cap S(y, c)\right|=m$. Since $\left|S\left(c^{\prime}\right)\right|=$ $\left|S\left(z, c^{\prime}\right)\right|=m$, we have $y \in S\left(c^{\prime}\right)$ by (ii). Thus for $x \in S\left(z, c^{\prime}\right) \cap S(y, c)$ 


$$
c^{\prime}=c^{\prime}+y=(x+z)+y=(x+y)+z=c+z=c .
$$

It follows that $|S(y, c)-S(z, c)|<m$. We can now proceed as in the proof of Theorem 2.1 to obtain our desired copy of $M_{m}$.

THEOREM 3.3. Suppose $L$ is a lattice of power $m$ with $m$ infinite and regular. Assume furthermore that $x, y \in L$ with $x \neq y$, that $x$ cannot be separated from $y$, and that $y$ cannot be separated from $x$. Then there is a sublattice of $L$ isomorphic to $M_{m}$ which has one of $x, y$ as the largest element and the other as the smallest.

Proof. We consider two cases.

Case 1. $x$ is not related to $y$ : First we note that $|S(x) \cup T(x)|<$ $m$. For otherwise $S(x) \cup T(x)$ separates $x$ from $y$. Now if $z \in L$ and $|S(z)|=m$ we must have $x \in S(z)$. For if not $S(z) \cup T(y)$ separates $y$ from $x$. Dually, if $|T(z)|=m$, then $x \in T(z)$. By Lemma 3.2 there is a copy of $M_{m}$ occuring as a sublattice of $L$ and having $x$ as an atom. If $\mathrm{y}$ does not belong to this sublattice then $x$ is separated from $y$. Otherwise we separate $x$ from $y$ by removing $y$ from this sublattice.

Case 2. $x$ is related to $y$ : Without loss of generality, assume that $x<y$. Furthermore, we assume that the conclusion of the theorem is false. We observe that if $z \in L$ with $|S(z)|=m$, then $x, y \in S(z)$. For if $x \notin S(z)$, then $S(z) \cup T(y)$ separates $y$ from $x$. If $y \notin S(z)$, then $S(z)$ separates $x$ from $y$. The dual argument gives $x, y \in T(w)$ whenever $|T(w)|=m$. We also note that $|S(x) \cup T(y)|<m$.

Suppose now that $|S(y) \cap T(x)|=m$. Applying Theorem 2.1 to the lattice $S(y) \cap T(x)$ gives

(i) $z \in L$ with $z<y$ and $|S(z) \cap T(x)|=m$,

(ii) $z \in L$ with $x<z$ and $|T(z) \cap S(y)|=m$, or (iii) there is a copy of $M_{m}$ occuring as a sublattice of $S(y) \cap T(x)$. Since none of these can happen, we must have $|S(y) \cap T(x)|<m$.

Assume now that $|T(x)|=m$. We know that

$$
|(T(x) \cap S(y)) \cup T(y)|<m .
$$

Also if $z \in T(x)$ with $|S(z) \cap T(x)|=m$, we have $y \in S(z) \cap T(x)$. If $z \in T(x)$ with $|T(z)|=m$, then $z=x$ and $y \in T(z)$. Hence we can apply Lemma 3.2 to the lattice $T(x)$ and the element $y$ to get a copy of $M_{m}$ occuring as a sublattice of $T(x)$ having $y$ as an atom. We must have $x$ as the smallest element of this sublattice. Thus if we remove $y$ from this sublattice, we separate $x$ from $y$. This shows that $|T(x)|<m$.

We now apply Lemma 3.2 to $L$ and the element $x$ to get a copy of $M_{m}$ having $x$ as an atom. If $y$ is not the largest element of this sublattice, then we have separated $x$ from $y$. If $y$ is the largest element of this sublattice, we just remove $x$ from this sublattice to 
separate $y$ from $x$. This final contradiction comes from the assumption that the conclusion of the theorem is false.

COROLLARY 3.4. In a lattice of power $m$ where $m$ is infinite and regular, there are at most two elements which do not lie in the complement of some sublattice of power $m$.

EXAMPLE 3.5. Let $k$ be any infinite cardinal. Take $L(k)$ to be the set of all finite subsets $S$ of $k$ which satisfy the restrictions

(i) if $|S|$ is odd, then $\frac{|S|-1}{2} \subseteq S$,

(ii) if $|S|$ is even, then $\frac{|S|}{2} \leqq S$.

If $S$ is a finite subset of $k$, we let $\bar{S}=S \cup n$ where $n$ is the smallest member of $\omega$ for which $S \cup n \in L(k)$. It is now fairly routine to check that $L(k)$, ordered by set inclusion, is a lattice in which the least upper bound of two elements $S$ and $T$ is $\overline{S \cup T}$, and the greatest lower bound is $S \cap T$.

It is clear that the atoms of this lattice are the sets of the form $\{\xi\}$ where $\xi \in k$. Also, we can check that any sublattice which contains infinitely many atoms contains each $n \in \omega$.

Suppose now that $m$ is an infinite cardinal with cf $(m)=k<m$. Then there are cardinals $m_{\alpha}<m$ for $\alpha \in k$ so that $\sum\left(m_{\alpha} \mid \alpha \in k\right)=m$. We obtain a lattice $L$ from the following diagram by letting the elements $\{\xi\}$ for $\xi \in k$ generate $L(k)$ as a sublattice:

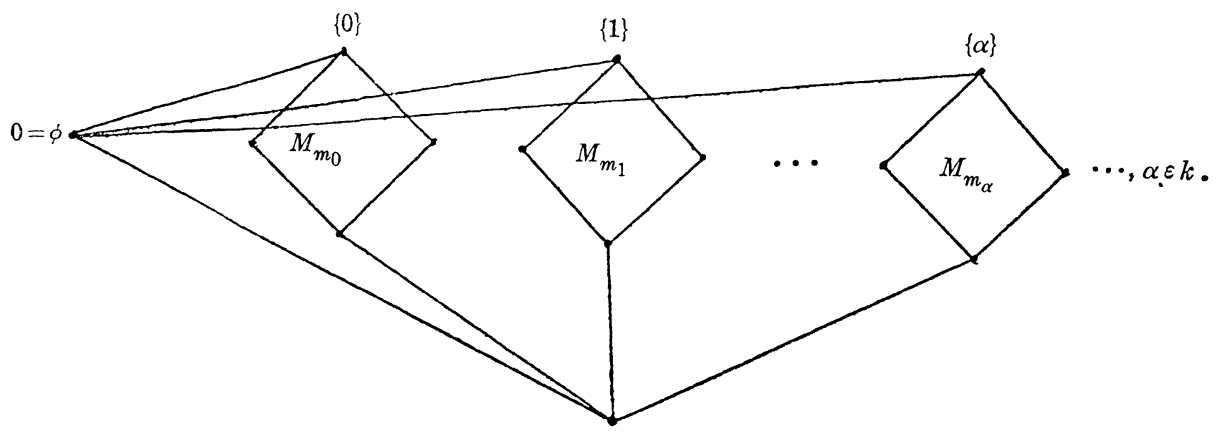

FiguRE 3

Then any sublattice of $L$ of power $m$ must contain infinitely many of the elements $\{\alpha\}$ with $\alpha \in k$. This shows that each $n \in \omega$ is in such a sublattice. Thus there are infinitely many elements of $L$ which lie in each sublattice of power $m$.

Lемма 3.6. If $L$ is a distributive lattice of power $m \geqq \omega$, then for any $y \in L$ we have $|S(y) \cup T(y)|=m$. 
Proof. Define a map $\phi$ of $L$ into $S(y) x T(y)$ by $\phi(x)=(x \cdot y, x+y)$. Since $L$ is distributive, $\phi$ is one-to-one (cf [1]). Hence $|S(y)| \cdot|T(y)|=$ $m$; so $|S(y)|=m$ or $|T(y)|=m$.

THEOREM 3.7. If $L$ is a distributive lattice of regular power $m \geqq w$, then each element of $L$ lies in the complement of some sublattice of power $m$.

Proof. Suppose $x_{0}$ is a member of each sublattice of power $m$. By Lemma 3.6 $\left|S\left(x_{0}\right) \cup T\left(x_{0}\right)\right|=m$. We assume that $\left|S\left(x_{0}\right)\right|=m$. Take any $x_{1} \in S\left(x_{0}\right)$ with $x_{1} \neq x_{0}$. Applying Lemma 3.6 to the lattice $S\left(x_{0}\right)$ and the element $x_{1}$ gives

$$
\left|S\left(x_{1}\right) \cup\left(T\left(x_{1}\right) \cap S\left(x_{0}\right)\right)\right|=m .
$$

Clearly we must have $\left|T\left(x_{1}\right) \cap S\left(x_{0}\right)\right|=m$. Let $Y$ be the set of all elements of $S\left(x_{0}\right)$ which are not related to $x_{1}$. Suppose $|Y|=m$. Then since

$$
Y=\bigcup\left\{T\left(x_{1}, z\right) \cap Y \mid z \in S\left(x_{1}\right)-\left\{x_{1}\right\}\right\},
$$

we must have $\left|T\left(x_{1}, z_{0}\right) \cap Y\right|=m$ for some $z_{0}<x_{1}$. Thus $\mid T\left(x_{1}, z_{0}\right) \cap$ $S\left(x_{0}\right) \mid=m$. However, since $L$ is distributive, $T\left(x_{1}, z_{0}\right) \cap S\left(x_{0}\right)$ is a sublattice of $L$ not containing $x_{0}$. Hence we must have $|Y|<m$ or $\left|S\left(x_{0}\right)-T\left(x_{1}\right)\right|<m$. This holds for any $x_{1}<x_{0}$.

Suppose $\xi<m$ and we have $\left\{x_{\beta} \mid \beta<\xi\right\}$ having the property that $1 \leqq \beta<\beta^{\prime}$ implies that $x_{\beta}<x_{\beta^{\prime}}<x_{0}$. Now

$$
\begin{aligned}
\mid S\left(x_{0}\right) & -\cap\left\{T\left(x_{\beta}\right) \mid \beta<\xi\right\} \mid \\
& =\left|\bigcup\left\{S\left(x_{0}\right)-T\left(x_{\beta}\right) \mid \beta<\xi\right\}\right| \\
& \leqq \sum\left(\left|S\left(x_{0}\right)-T\left(x_{\beta}\right)\right| ; \beta<\xi\right) \\
& <m
\end{aligned}
$$

since $m$ is regular. We take $x_{\xi} \in \cap\left\{S\left(x_{0}\right) \cap T\left(x_{\beta}\right) \mid \beta<\xi\right\}, x_{\xi}<x_{0}$. In this way we get $\left\{x_{\xi} \mid \xi<m\right\}$ a chain of elements of $L$. But then $\left\{x_{\xi} \mid 1 \leqq \xi<m\right\}$ is a sublattice of $L$ not containing $x_{0}$. This contradiction completes the proof.

\section{BIBLIOGRAPHY}

1. G. Birkhoff, Lattice theory, Vol. XXV; American Mathematical Society Colloquium Publications, 3rd ed., Providence; American Mathematical Society, 1967.

2. P. Erdös and A. Hajnal, On a problem of B. Jónsson, Bulletin de L'Academie Polonaise de Sciences, 14 (1966), 19-23.

3. H. J. Keisler and F. Rowbottom, Constructible sets and weakly compact cardinals, Notices of the American Mathematical Society 12 (1965), 373-374. 
4. A. Kostinsky, Recent results on Jónsson algebras. Seminar notes, Department of Mathematics, University of California at Berkeley, 1966.

5. S. P. Strunkov, Subgroups of periodic groups, Soviet-Mathematics-Doklady 7 (1966), 1201-1203.

6. T. Whaley, Algebras satisfying the descending chain condition for subalgebras, Vanderbilt University Dissertation, 1968.

7. - Infinite algebras satisfying the descending chain condition, Notices of the American Mathematical Society 14 (1967), 714.

Received June 24, 1968. This work was supported in part by NSF Grant GP 7252. These results are taken from the author's doctoral thesis, supervised by Professor B. Jónsson at Vanderbilt University.

VANDERBILT UNIVERSITY AND

FISK UNIVERSITY 


\section{PACIFIC JOURNAL OF MATHEMATICS}

\section{EDITORS}

H. ROYDEN

Stanford University

Stanford, California

\section{R. $R$ PHELPS}

University of Washington

Seattle, Washington 98105

\section{J. DugunduI}

Department of Mathematics

University of Southern California

Los Angeles, California 90007

RICHARD ARENS

University of California

Los Angeles, California 90024

\section{ASSOCIATE EDITORS}

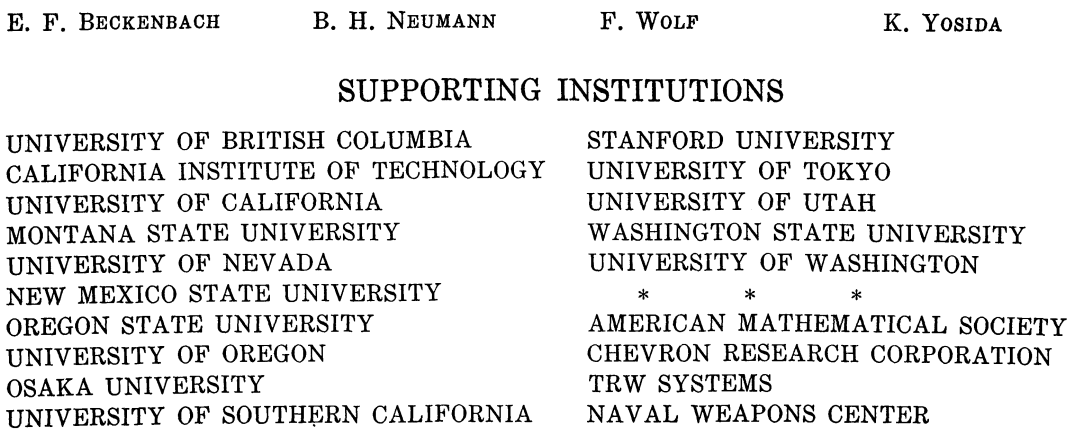

The Supporting Institutions listed above contribute to the cost of publication of this Journal, but they are not owners or publishers and have no responsibility for its content or policies.

Mathematical papers intended for publication in the Pacific Journal of Mathematics should be in typed form or offset-reproduced, double spaced with large margins. Underline Greek letters in red, German in green, and script in blue. The first paragraph or two must be capable of being used separately as a synopsis of the entire paper. It should not contain references to the bibliography. Manuscripts, in duplicate if possible, may be sent to any one of the four editors. Please classify according to the scheme of Math. Rev. 36, 1539-1546. All other communications to the editors should be addressed to the managing editor, Richard Arens, University of California, Los Angeles, California, 90024.

50 reprints are provided free for each article; additional copies may be obtained at cost in multiples of 50 .

The Pacific Journal of Mathematics is published monthly. Effective with Volume 16 the price per volume (3 numbers) is $\$ 8.00$; single issues, $\$ 3.00$. Special price for current issues to individual faculty members of supporting institutions and to individual members of the American Mathematical Society: $\$ 4.00$ per volume; single issues $\$ 1.50$. Back numbers are available.

Subscriptions, orders for back numbers, and changes of address should be sent to Pacific Journal of Mathematics, 103 Highland Boulevard, Berkeley, California, 94708.

PUBLISHED BY PACIFIC JOURNAL OF MATHEMATICS, A NON-PROFIT CORPORATION

Printed at Kokusai Bunken Insatsusha (International Academic Printing Co., Ltd.), 7-17, Fujimi 2-chome, Chiyoda-ku, Tokyo, Japan. 


\section{Pacific Journal of Mathematics}

Vol. 28, No. $2 \quad$ April, 1969

Richard Arens and Donald George Babbitt, The geometry of relativistic

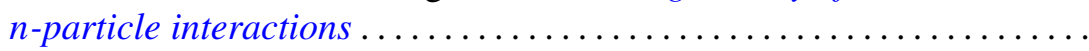

Kirby Alan Baker, Hypotopological spaces and their embeddings in lattices

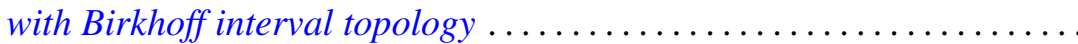

J. Lennart (John) Berggren, Finite groups in which every element is conjugate to its inverse ........................... 289

Beverly L. Brechner, Homeomorphism groups of dendrons . . . . . . . . . . . 295

Robert Ray Colby and Edgar Andrews Rutter, QF - 3 rings with zero singular ideal ................................. 303

Stephen Daniel Comer, Classes without the amalgamation property....... 309

Stephen D. Fisher, Bounded approximation by rational functions ......... 319

Robert Gaines, Continuous dependence for two-point boundary value problems..................................... 327

Bernard Russel Gelbaum, Banach algebra bundles ............... 337

Moses Glasner and Richard Emanuel Katz, Function-theoretic degeneracy criteria for Riemannian manifolds ...................... 351

Fletcher Gross, Fixed-point-free operator groups of order $8 \ldots \ldots \ldots \ldots 357$

Sav Roman Harasymiv, On approximation by dilations of distributions . . . . 363

Cheong Seng Hoo, Nilpotency class of a map and Stasheff's criterion ... . . 375

Richard Emanuel Katz, A note on extremal length and modutus.......... 381

H. L. Krall and I. M. Sheffer, Difference equations for some orthogonal polynomials ................................

Yu-Lee Lee, On the construction of lower radical properties ........... 393

Robert Phillips, Liouville's theorem........................... 397

Yum-Tong Siu, Analytic sheaf cohomology groups of dimension $n$ of

n-dimensional noncompact complex manifolds ..... . .

Michael Samuel Skaff, Vector valued Orlicz spaces. II...

James DeWitt Stein, Homomorphisms of $B^{*}$-algebras .... . .

Mark Lawrence Teply, Torsionfree injective modules .... . . .

Richard R. Tucker, The $\delta^{2}$-process and related topics. II .

David William Walkup and Roger Jean-Baptiste Robert Wets, Lifting

projections of convex polyhedra...

Thomas Paul Whaley, Large sublattices of a lattice. 